\title{
The spin-one Heisenberg-biquadratic quantum spin chain treated by the coupled-cluster method
}

\author{
R F Bishop, J B Parkinson and Yang Xian \\ Department of Mathematics, UMIST, PO Box 88, Manchester M60 JQD, UK
}

Received 16 August 1993

\begin{abstract}
The coupled-cluster method is used to obtain the ground-state energy of the isotropic Heisenberg-biquadratic quantum spin-one chain as a function of the ratio of the magnitudes of the two terms in the Hamiltonian. Two different model states are used which are expected to be valid in different regimes. In both cases we use simple approximation schemes to obtain numerical results for the ground-state energy which are compared with results of exact diagonalizations of short chains. For both cases we are able to incorporate some of the long-range correlations explicitly, using the so-called full-suB2 approximation schemes, and this leads to evidence of phase changes at certain points. These are discussed in the light of known and conjectured phase transitions in this system.
\end{abstract}

\section{Introduction}

Following the initial work of Roger and Hetherington (1990) we have shown in a recent series of papers (Bishop et al 1991a, b, 1992a, b) that the coupled-cluster method (CCM) is a powerful tool for treating quantum spin systems. We shall refer to the 1991b paper as I. (For introductory reviews of the CCM applied to a wide variety of quantum many-body problems see Bishop and Kümmel 1987 and Bishop 1991.) The main advantages of the CCM in principle are that it is an $a b$ initio method and that it is completely systematic. In practice we have shown that for antiferromagnetic quantum spin systems it can give useful numerical results: even in rather low orders of approximation the results for ground-state energies are encouraging when compared either with exact results (for example for the $s=1 / 2 X X Z$ chain) or with direct numerical results obtained from short-chain extrapolations when exact results are not known.

In this paper we shall use the method on the $s=1$ isotropic Heisenberg-biquadratic chain described by the Hamiltonian

$$
\mathcal{H}=\frac{1}{2} \sum_{l} \sum_{\rho}\left(\cos \omega\left(s_{l} \cdot s_{l+\rho}\right)+\sin \omega\left(s_{l} \cdot s_{l+\rho}\right)^{2}\right)
$$

where the sum over $l$ is over all $N$ atoms with periodic boundary conditions, and the sum over $\rho$ is over nearest neighbours $(\rho= \pm 1)$. We shall also use a notation in which $\cos \omega=J_{1}$ and $\sin \omega=J_{2}$. This model has an interesting zero-temperature phase diagram as a function of $\omega$, which is shown in figure 1 . Within the non-ferromagnetic regime $-3 \pi / 4<\omega<\pi / 2$, the system is integrable using the Bethe ansatz (Bethe 1931) at the points marked $T$, at $\omega=-\pi / 4$ (Takhtajan 1982, Babujian 1982), and $S$, at $\omega=\pi / 4$ (Lai 1974, Sutherland 1975). The exact ground state is also known at the point marked B, at $\omega=-\pi / 2$ (Parkinson 1987, 1988, Barber and Bachelor 1989, Klümper 1989, 1990) and 


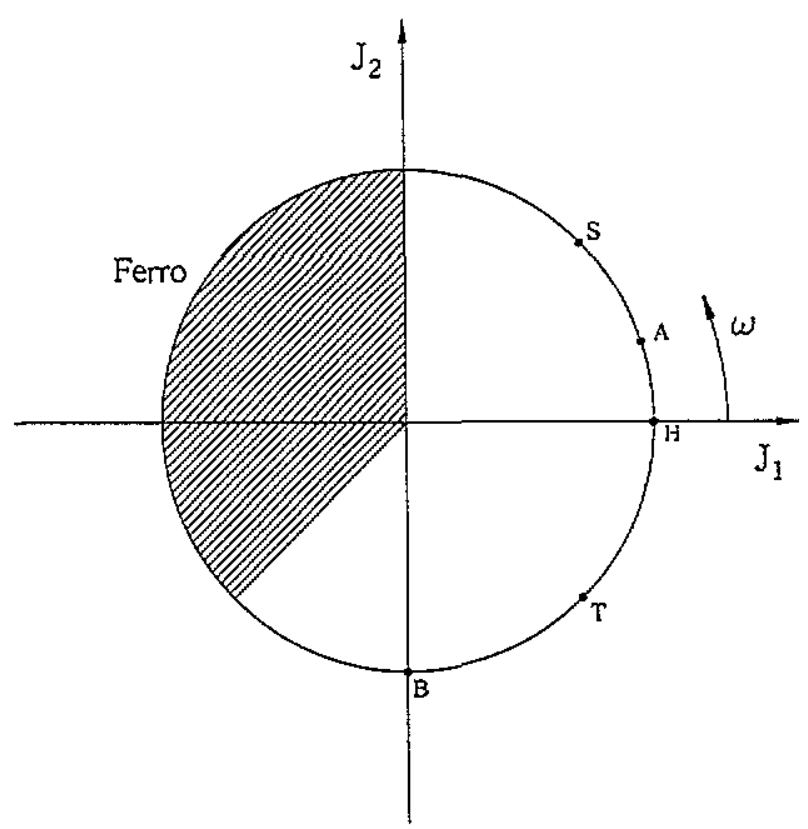

Figure 1. The zero-temperature phase diagram of the $s=1$ Heisenberg-biquadratic quantum spin chain. The various marked points, referred to in the text, correspond to values of $\omega$ at which exact results are known.

at the point marked $A$, at $\omega=\tan ^{-1}$ (1/3) (Affleck et al 1987, 1988). The pure Heisenberg point marked $\mathrm{H}$, at $\omega=0$, was discussed by Haldane (1983a, b) who predicted the existence of a previously unexpected gap.

The system is now believed to have a doubly degenerate ground state and a gap in the whole of the region $-3 \pi / 4<\omega<-\pi / 4$. The nature of the ground state in this region is complex with 'dimerized' and 'spin-nematic' phases possibly present (see Chubukov 1991 for a discussion). At the integrable point $\omega=-\pi / 4$ there is a transition to a Haldane-like phase with a singly degenerate ground state and a gap, which continues to $\omega>0$ and probably up to $\omega=\pi / 4$, including the $\omega=\tan ^{-1}(1 / 3)$ point. Again, this regime has a twofold real-space periodicity. The threefold real-space symmetry of the exact solution at $\omega=\pi / 4$ is believed to exist over the whole of the region $\pi / 4 \leqslant \omega<\pi / 2$ and this region also appears to be gapless (Fáth and Sólyom 1991, 1993). Chubukov (1990) has predicted unusual ordering in this region and also in the region $-3 \pi / 4<\omega \lesssim-\pi / 2$. Other recent numerical work on this system includes that of Xiang and Gehring (1993), who used a truncated basis expansion inspired by the Wilson renormalization group method in real space.

The CCM should be a good candidate for study of this system with its mixture of exact and non-exact results and rich phase structure. This is because the CCM is applicable equally to integrable and non-integrable systems and also has the potential to predict phase changes in a way that purely numerical results cannot.

Finally we note that $\mathrm{CCM}$ results for the pure Heisenberg model, $\omega=0$, were given in our earlier paper (Bishop et al 1992a). 


\section{The CCM based on the Nél model state}

The starting point of the CCM is the choice of an 'uncorrelated' model or reference state $|\Phi\rangle$. One obvious choice for $|\Phi\rangle$ is the usual two-sublattice Neel state, $|\Phi\rangle \rightarrow|\mathrm{N} 2\rangle=$ $1+-+-+-+\ldots\rangle$, where + denotes an $s=1$ atom in a state with $s^{2}=+1$ and denotes a state with $s^{z}=-1$. We point out that, unlike the case for $s=1 / 2$ systems, the choice $s^{z}=0$ is a possibility and we shall consider model states in which this occurs later. If we take the expectation value of $\mathcal{H}$ given by (1.1) in $|\mathrm{N} 2\rangle$, we obtain for the ground state energy per spin

$$
\langle\mathrm{N} 2|\mathcal{H}| \mathrm{N} 2\rangle / N=-\cos \omega+2 \sin \omega=-J_{1}+2 J_{2}
$$

The factor of two in the second term is already a quantum effect.

We use the usual spin angular momentum operators $s^{\alpha}, \alpha=x, y, z$, and $s^{ \pm}=s^{x} \pm$ is $y$. Following Roger and Hetherington (1990), for ease of later description we perform a notional rotation of $180^{\circ}$ on one sublattice so that the model state may be referred to as having all spins pointing down. This means that a 'creation' operator with respect to the model state $|\mathrm{N} 2\rangle$ will always be a raising operator $s^{+}$even on the 'up' sublattice. The term $s_{l} \cdot s_{l+\rho}$ in the Hamiltonian now becomes

$$
s_{l} \cdot s_{l+\rho}=-\left[s_{l}^{z} s_{l+\rho}^{z}+1 / 2\left(s_{l}^{+} s_{l+\rho}^{+}+s_{l}^{-} s_{l+\rho}^{-}\right)\right]
$$

In the CCM the true ground state is written

$$
|\Psi\rangle=\mathrm{e}^{5}|\Phi\rangle
$$

The CCM correlation operator $S$ is constructed entirely out of creation operators with respect to the model state, i.e., out of a sum of terms containing all possible combinations of the $\left\{s_{l}^{+}\right\}$creation operators consistent with the conserved quantities. For the ground state of (1.1) we require that $s_{\mathrm{T}}^{z} \equiv \sum_{l} s_{l}^{2}=0$. Any particular approximation consists of selecting a subset of these terms.

\subsection{The SUB2 approximation}

We shall first consider the approximation known as full suB2. This includes explicitly all possible correlations involving two spin-raising operators. No other correlations are included in the correlation operator $S$ explicitly although the CCM generates many higherorder correlations automatically from the lower-order ones, due to the exponential structure of (2.3). In this approximation we write

$$
S \rightarrow S_{\mathrm{SUB} 2}=(1 / 2) \sum_{i} \sum_{r} b_{r} s_{i}^{+} s_{i+r}^{+}
$$

where $i$ runs over all $N$ sites and $r$ is any positive or negative odd integer.

Operators are normally required in similarity transformed form, $\tilde{A} \equiv \mathrm{e}^{-S} A \mathrm{e}^{S}$. Using the usual expansion in terms of nested commutators,

$$
\mathrm{e}^{-S} A \mathrm{e}^{S}=A+[A, S]+\frac{1}{2}[[A, S], S]+\cdots
$$


then $\tilde{s}_{l}^{+}=s_{l}^{+} ; \tilde{s}_{l}^{z}=s_{l}^{z}+\left[s_{l}^{z}, S\right] ; \tilde{s}_{l}^{-}=s_{l}^{-}+\left[s_{l}^{-}, S\right]+\frac{1}{2}\left[\left[s_{l}^{-}, S\right], S\right]$. These expressions for $\tilde{s}_{l}^{+}, \tilde{s}_{l}^{2}$, and $\tilde{s}_{l}^{-}$are exact for any $S$ since $S$ contains only creation operators, i.e., $\left\{s_{i}^{+}\right\}$. For the particular choice given by $(2.4)$ we obtain

$$
\begin{aligned}
& \tilde{s}_{l}^{+}=s_{l}^{+} \\
& \tilde{s}_{l}^{z}=s_{l}^{z}+\sum_{r} b_{r} s_{l}^{+} s_{l+r}^{+} \\
& \tilde{s}_{l}^{-}=s_{l}^{-}-2 \sum_{r} b_{r} s_{l}^{z} s_{l+r}^{+}-\sum_{r} \sum_{l} b_{r} b_{l} s_{l}^{+} s_{l+r}^{+} s_{l+t}^{+}
\end{aligned}
$$

and the indices $r$ and $t$ are again any positive or negative odd integers.

It is useful to split up the $\tilde{\mathbf{s}}_{l} \cdot \overline{\mathbf{s}}_{l+\rho}$ term which occurs in the similarity transformed Hamiltonian into separate parts, each of which corresponds to a given total change in the $z$ component of angular momentum. Hence we write

$$
\tilde{s}_{l} \cdot \tilde{s}_{l+\rho}=\sum_{n=-2}^{6} X_{n}
$$

where $n$ is an even integer corresponding to the net change. The expressions for these $X_{n}$ operators are given in appendix 1.

Using (2.3) together with the Schrödinger equation

$$
\mathcal{H}|\Psi\rangle=E_{\mathrm{g}}|\Psi\rangle
$$

yields

$$
E_{\mathrm{g}}=\{\mathrm{N} 2|\tilde{\mathcal{H}}| \mathrm{N} 2\rangle .
$$

For the first term in the Hamiltonian it is only necessary to calculate $\left(\mathrm{N} 2\left|X_{0}\right| \mathrm{N} 2\right\rangle$ before summing over $l$ and $\rho$, but the second term requires $\left\{\mathrm{N} 2\left|\left\{X_{0} X_{0}+X_{-2} X_{2}\right\}\right| \mathrm{N} 2\right\}$. (Note that $X_{-2}|\mathrm{~N} 2\rangle=0$.) The result for this approximation scheme is

$$
E_{\mathrm{g}} / N=-J_{1}\left(1+2 b_{1}\right)+J_{2}\left(2+2 b_{1}+2 b_{1}^{2}\right) \text {. }
$$

The fact that only the $b_{1}$ coefficient occurs is a reflection of the nearest-neighbour interaction form of $\mathcal{H}$.

A set of coupled non-linear equations for the coefficients $b_{r}$ is obtained by operating on both sides of (2.8), again using (2.3), with

$$
\sum_{i}\left(\mathrm{~N} 2 \mid s_{i}^{-} s_{i+t}^{-} \mathrm{e}^{-s}\right. \text {. }
$$

Since this operator is clearly orthogonal to $|\Psi\rangle=\exp (S)|\mathrm{N} 2\rangle$, the right-hand side of the resulting equation is zero. On the left-hand side we need only retain terms in $\tilde{\mathcal{H}}|\mathrm{N} 2\rangle$ which involve precisely 2 spin flips. For the first term in $\mathcal{H}$ this involves the sum over $l$ and $\rho$ of $X_{2}|\mathrm{~N} 2\rangle$ while for the second term we need the sum of terms involving $X_{2} X_{0}|\mathrm{~N} 2\rangle$, $X_{0} X_{2}|\mathrm{~N} 2\rangle$ and $X_{-2} X_{4}|\mathrm{~N} 2\rangle$.

After considerable algebra the following set of equations results:

$$
A \sum_{\rho} \delta_{t \rho}+B b_{t}+C \sum_{r} \sum_{\rho} b_{t+\rho+r} b_{r}=0
$$

where

$$
\begin{aligned}
& A=-J_{1}\left(1+2 b_{1}+2 b_{1}^{2}\right)+J_{2}\left(1+2 b_{1}\right)\left(1+2 b_{1}+6 b_{1}^{2}\right) \\
& B=8 J_{1}\left(1+2 b_{1}\right)-8 J_{2}\left(1+4 b_{1}+6 b_{1}^{2}\right) \\
& C=-4 J_{1}+4 J_{2}\left(1+2 b_{1}\right) .
\end{aligned}
$$

In these equations $\rho= \pm 1$, and $r$ and $t$ may be any positive or negative odd integers. 


\subsection{The SUB2-2 scheme}

Before solving the full suB2 equations (2.11) it is interesting to consider a much simpler approximation, known as SUB2-2, in which all the coefficients $\left\{b_{t}\right\}$ are set equal to zero except $b_{ \pm 1}$, with $b_{-1}=b_{1}$. In this case we obtain the single equation

$$
-J_{1}\left(1-6 b_{1}-2 b_{1}^{2}\right)+J_{2}\left(1-4 b_{1}-10 b_{1}^{2}-12 b_{1}^{3}\right)=0
$$

which is easily solved for any value of $\omega$, i.e. of $J_{1}$ and $J_{2}$.

The results of this approximation are shown in figure 2, where they are compared with results obtained by numerical diagonalization of short chains. The $N \rightarrow \infty$ limit is obtained by extrapolating the plot of $E_{\mathrm{g}} / N$ against $N^{-2}$ for $N \leqslant 14$. The resulting curve is labelled 'exact' in the figure and is expected to be accurate to within the resolution of the figure. For this reason we have not pursued these short-chain calculations to higher $N$ as was done, for example, by Sakai and Takahashi (1991) for the case $\omega=0$.

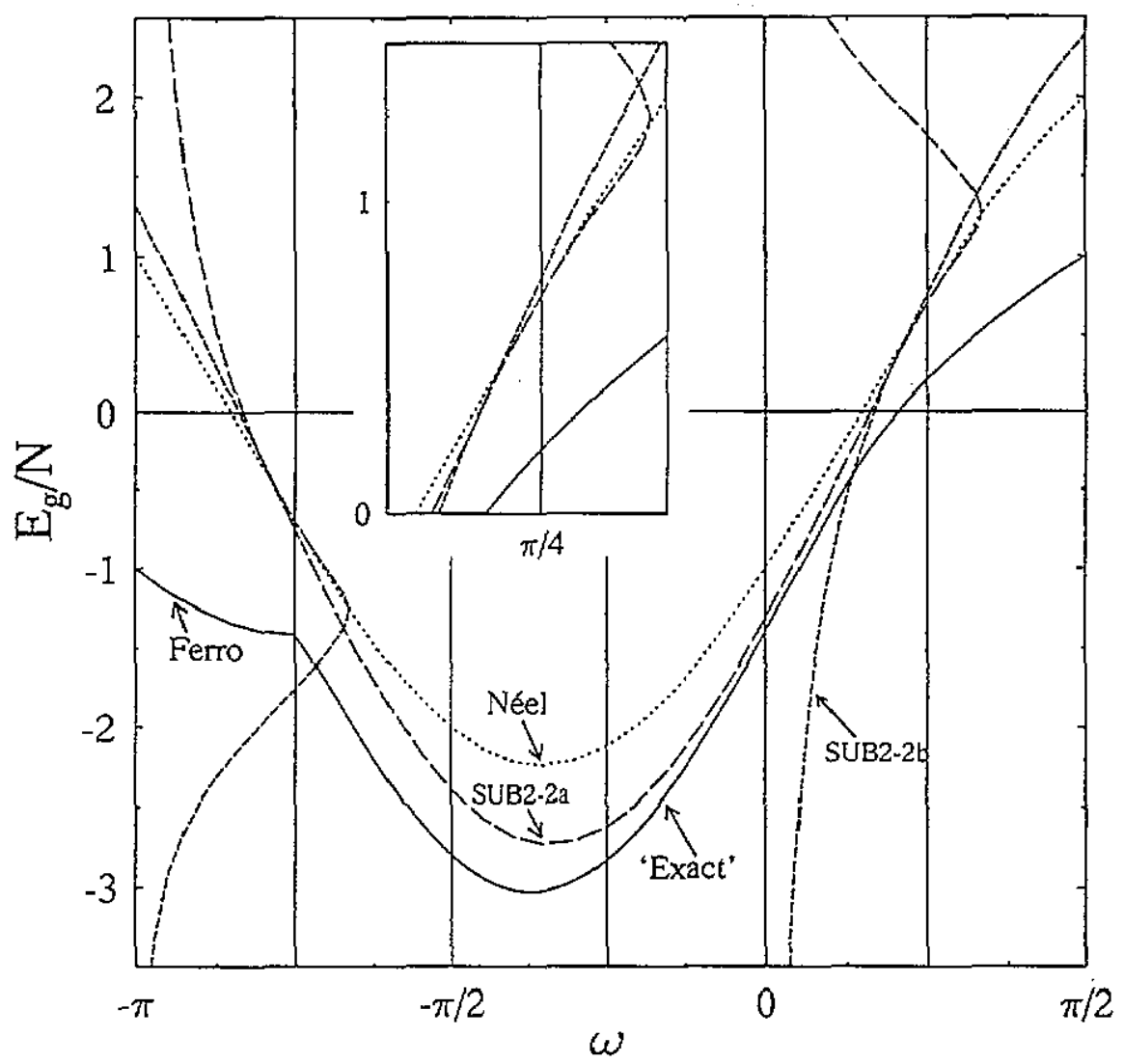

Figure 2. Ground-state energy per spin for the suB2-2 approximation scheme based on the Neel model state. The two continuous parts of the solution, SUB2-2a and SUB2-2b are indicated by long and short dashed lines respectively. The dotted line (marked Neel) is the expectation vaiue of $\mathcal{K} / N$ in the model state $[\mathrm{N} 2\}$. The solid line (marked 'exact') is the exact value in the ferromagnetic region shown, $\omega \leqslant-3 \pi / 4$, and a numerical estimate based on extrapolated short chain calculations elsewhere, as discussed in the text. 
For the SUB2-2 approximation we find that in the regions $-2.0866<\omega<-0.0155$ and $1.0550<\omega<3.1261$ there is only one real solution: elsewhere there are three. However it is more convenient to consider the solutions as lying on two distinct branches. The first of these, which diverges to $+\infty$ at $\omega=-\pi, 0$ we refer to as SUB2-2a, while the one that diverges to $-\infty$ at these points we refer to as SUB2-2b. The two branches cross close to $\omega=\pi / 4,-3 \pi / 4$.

The SUB2-2a branch lies closest to the 'exact' result for $\omega$ in the vicinity of 0 , but provides a reasonable approximation over much of the region $-3 \pi / 4 \leqslant \omega \leqslant \pi / 4$. The SUB2-2b branch is related to the SUB2-2a one by the transformation $\omega \rightarrow \omega+\pi, E_{\mathrm{g}} \rightarrow-E_{\mathrm{g}}$, since $\omega \rightarrow \omega+\pi$ is equivalent to $J_{1} \rightarrow-J_{1}, J_{2} \rightarrow-J_{2}$. This second branch would obviously be an approximation to the highest state in its region of existence. The other notable feature of the SUB2-2a branch is the point $\omega=1.0550, E_{g} / N=1.2490$ at which the SUB2-2a curve tums over. We believe this is a precursor of a phase transition which is indicated more clearly in the full SUB2 solution described in the next section.

\subsection{Solution of the full SUB2 equations}

Equations (2.11) can be solved by Fourier transform in the manner described in our earlier paper (I). The result is

$$
b_{r}=-\frac{B}{4 C} \frac{1}{\pi} \int_{0}^{\pi} \mathrm{d} q \frac{\cos r q}{\cos q}\left[1 \pm \sqrt{1-\kappa \cos ^{2} q}\right]
$$

where $\kappa \equiv 16 A C / B^{2}$. The choice $r=1$ in (2.14) gives a self-consistency equation for $b_{1}$ which can be solved numerically. Both choices of sign of the square root are needed for a complete solution.

Using the numerical result for $b_{1}$ together with (2.10) we obtain the ground-state energy per spin in the full SUB2 approximation scheme as a function of $\omega$. We obtain two solutions, labelled SUB2a and SUB2b in figure 3, which are similar in many respects to the SUB2-2a and suB2-2b solutions. In fact the results are numerically very close over much of the region.

One interesting feature of the full sUB2 calculation for the $s=1 / 2 X X Z$ model was the existence of a terminating point which we argued corresponded to a phase change in the system for a particular value of the anisotropy parameter. An equation very similar to (2.14) was also found in the $s=1 / 2$ model, and the terminating point occurred when $\kappa=1$ as values greater than 1 would clearly involve complex solutions of (2.14).

In the present case, terminating points with $\kappa=1$, marked $\mathrm{A}$ and $\mathrm{B}$ in the figure, were found for SUB2a at $\omega=0.9864, E_{\mathrm{g}} / N=1.1545$ and $\omega=0.1486, E_{\mathrm{g}} / N=1.2142$. (Corresponding terminating points for the sUB2b solution also exist, of course.) Between these two points there is no real solution, leading to the gap in the curve shown on the figure. There is a significant difference between the SUB2-2a and SUB2a solutions close to this terminating point and this is shown on the enlarged portion of the figure. Again this behaviour is strongly reminiscent of the $X X Z$ model. The disconnected part of the suB2a curve also differs significantly from the SUB2-2a curve, but the solution here is physically unrealistic anyway.

The terminating point $A$ may well correspond to the transition at $\omega=\pi / 4$ to a phase with different real-space periodicity.

\section{The CCM based on other model states}

\subsection{Alternative model states}

In the CCM method one requires both a model state and a complete set of creation operators 


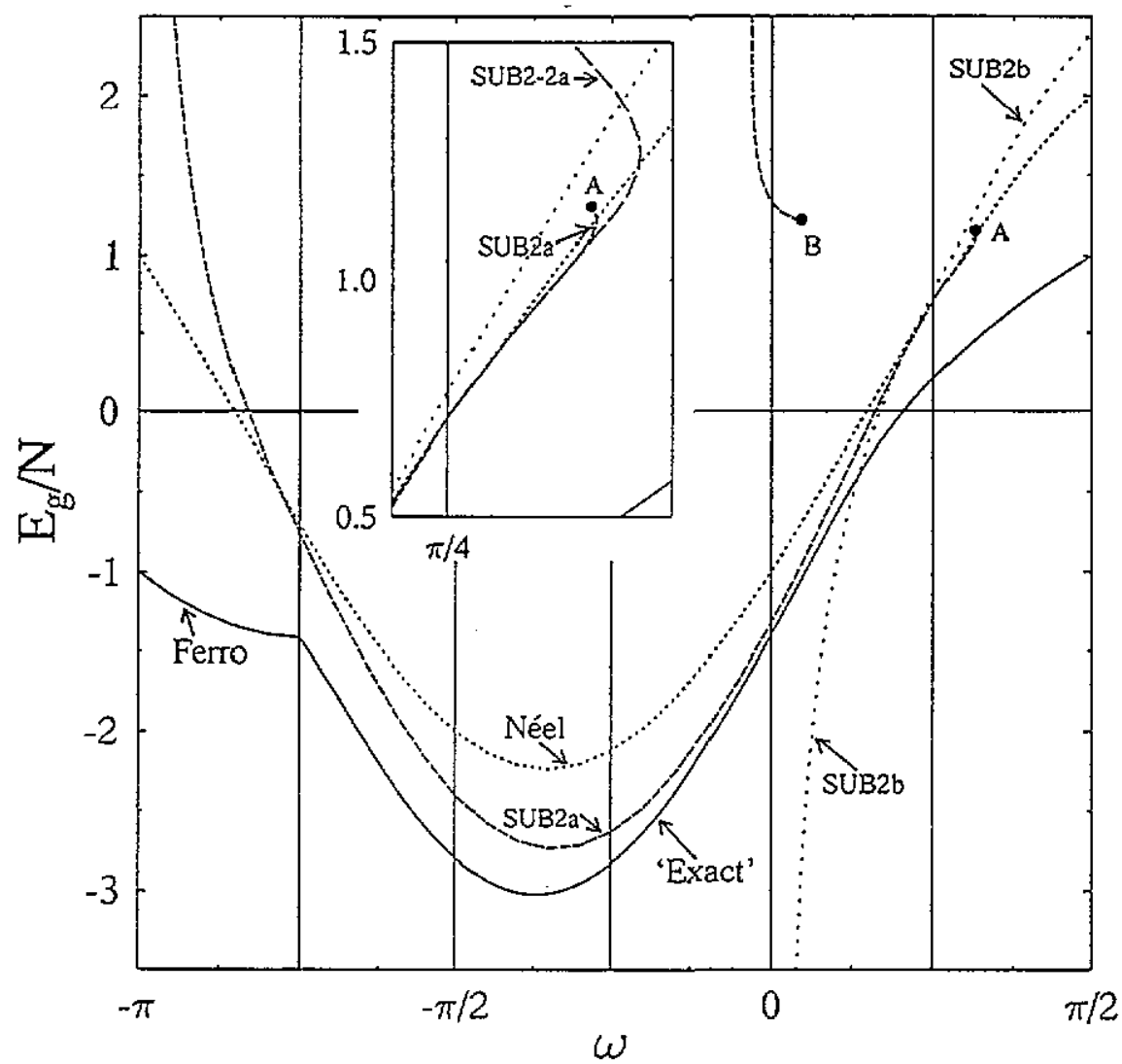

Figure 3. Ground-state energy per spin for the full SUB2 approximation scheme based on the Neel model state, using the notation of figure 2. The insert is an enlargement of the region close to the terminating point $A$, and also shows the suB2-2a solution for comparison. For clarity, only the portion of the SUB2b solution which lies between $\omega=0$ and $\omega=\pi / 2$ is shown.

with respect to the model state from which all other states can be constructed. For an antiferromagnetic spin-1/2 system it is difficult to see any alternative to the Néel state, equivalent to the $|\mathrm{N} 2\rangle$ state used in the previous sections. However for an $s=1$ system there are clearly other possible model states. We discuss in this section some of these, and for one in particular obtain a suitable complete set of creation operators.

The ground state of (1.1) for $-3 \pi / 4<\omega<\pi / 2$ lies in the sector $s_{T}^{z}=0$. Any model state which is an eigenstate of all the individual $s_{i}^{z}$ operators thus requires an equal number of atoms with $s_{i}^{2}=+1$ and -1 . However there may be an arbitrary number of atoms with $s_{i}^{z}=0$. We have considered three such model states as follows,

$$
\begin{aligned}
& |\mathrm{N} 1\rangle \equiv|0,0,0,0,0,0,0,0,0, \ldots\rangle \\
& |\mathrm{N} 3\rangle \equiv|+, 0,-,+, 0,-,+, 0,-, \ldots\rangle \\
& \mid \mathrm{N} 4\} \equiv|+, 0,-, 0,+, 0,-, 0,+, \ldots\rangle
\end{aligned}
$$

We refer to the first of these as the 'planar' model state. It is motivated by a suggestion by Chubukov (1990) that there may exist a 'planar quadrupolar' phase in the region $\omega$ close 
to but greater than $-3 \pi / 4$. The second, which clearly has threefold spatial symmetry, is motivated by the exact result at $\omega=/ \pi / 4$ which is known to have this symmetry and the recent numerical studies of Fath and Sólyom $(1991,1993)$ and Xiang and Gehring (1993) confirming the threefold symmetry over the region $\pi / 4 \leqslant \omega<\pi / 2$. The third, with fourfold symmetry, will be discussed shortly.

If we calculate the expectation value of the Hamiltonian (1.1) in the Néel state $|\mathrm{N} 2\rangle$, each of the above states, and the completely aligned ferromagnetic state $|F\rangle$ we obtain

$$
\begin{aligned}
& \langle\mathrm{N} 2|\mathcal{H}| \mathrm{N} 2\rangle=N\left(-J_{1}+2 J_{2}\right) \\
& \langle\mathrm{N} 1|\mathcal{H}| \mathrm{N} 1\rangle=2 N J_{2} \\
& \langle\mathrm{~N} 3|\mathcal{H}| \mathrm{N} 3\rangle=N\left(-J_{1}+4 J_{2}\right) / 3 \\
& \langle\mathrm{~N} 4|\mathcal{H}| \mathrm{N} 4\rangle=N J_{2} \\
& \langle\mathrm{~F}|\mathcal{H}| \mathrm{F}\rangle=N\left(J_{1}+J_{2}\right) .
\end{aligned}
$$

These expectation values are plotted as functions of $\omega$ in figure 4 . The ferromagnetic state gives the lowest expectation value in the region $\pi / 2<\omega<5 \pi / 4$ which is believed to be the actual ferromagnetic region. The Néel state $|\mathrm{N} 2\rangle$, with twofold symmetry, has the lowest expectation value in the region $-\pi / 2<\omega<\pi / 4$. For $\pi / 4<\omega<\pi / 2$ the state $|\mathrm{N} 4\rangle$ has a lower expectation value than either $|\mathrm{N} 2\rangle$ or $\mid \mathrm{N} 3\}$. In this region the true ground state is believed to have threefold symmetry, and one might have expected $|\mathbf{N} 3\rangle$ to be lowest, suggesting that these very simple model states are not very accurate. Nevertheless, the results do at least agree with the fact that the twofold perodicity of the Neel state is not present in this region. Finally in the region $-3 \pi / 4<\omega<-\pi / 2$ the planar state $|N 1\rangle$ has the lowest expectation value of these simple model states.

We shall not consider the possible model states $|\mathrm{N} 3\rangle$ or $|\mathrm{N} 4\rangle$ further in this paper. Instead we shall develop a CCM formalism for the planar model state $|\Phi\rangle \rightarrow|\mathrm{N} 1\rangle$, with the a priori expectation that it will be most relevant in the region $-3 \pi / 4<\omega<-\pi / 2$.

Finally, in figure 4 we also show the results of calculations based on a dimerized state (Chubukov 1991) and a trimerized state (Xian 1993). The dimerized (or spin-Peierls) state $|D\rangle$ is given by a sequence of spin-singlet states formed from adjacent spins on the chain, while the trimerized state $|T\rangle$ is similar to the state $|D\rangle$ but with each singlet state formed from three adjacent spins. Both states thus have a simple valence-bond interpretation (and see Xian 1993 for further details). It is straightforward to show that the energy expectation values for the Hamiltonian of (1.1) in these two model states are given by $\langle\mathrm{D}|\mathcal{H}| \mathrm{D}\rangle=N\left(-J_{1}+8 J_{2} / 3\right)$ and $\langle\mathrm{T}|\mathcal{H}| \mathrm{T}\rangle=N\left(-6 J_{1}+10 J_{2}\right) / 9$

It can clearly be seen that in the regions where the dimerized and trimerized phases are believed to exist, the above simple estimates generally lie closer to the exact ground-state energy than the corresponding estimates based on the states $\mid \mathrm{N} 1\}$ and $|\mathrm{N} 2\rangle$. It would thus be of particular interest to attempt similar CCM analyses to those performed here, but based on the state $|D\rangle$ or $|T\rangle$ as model state. Although such calculations are undoubtedly of greater algebraic and computational complexity than those discussed here, we hope to report results in the near future.

\subsection{Operators for the planar model state}

The normal raising and lowering operators $s_{i}^{ \pm}$cannot be used for a CCM formalism based on $|\Phi\rangle \rightarrow|N|\}$. The reason for this is that to create a state $|+\rangle$ from $|0\rangle$ needs operator $s_{i}^{+}$ while to create a state $|-\rangle$ from $|0\rangle$ needs $s_{i}^{-}$. Clearly $s_{i}^{-}$also acts as a destruction operator 


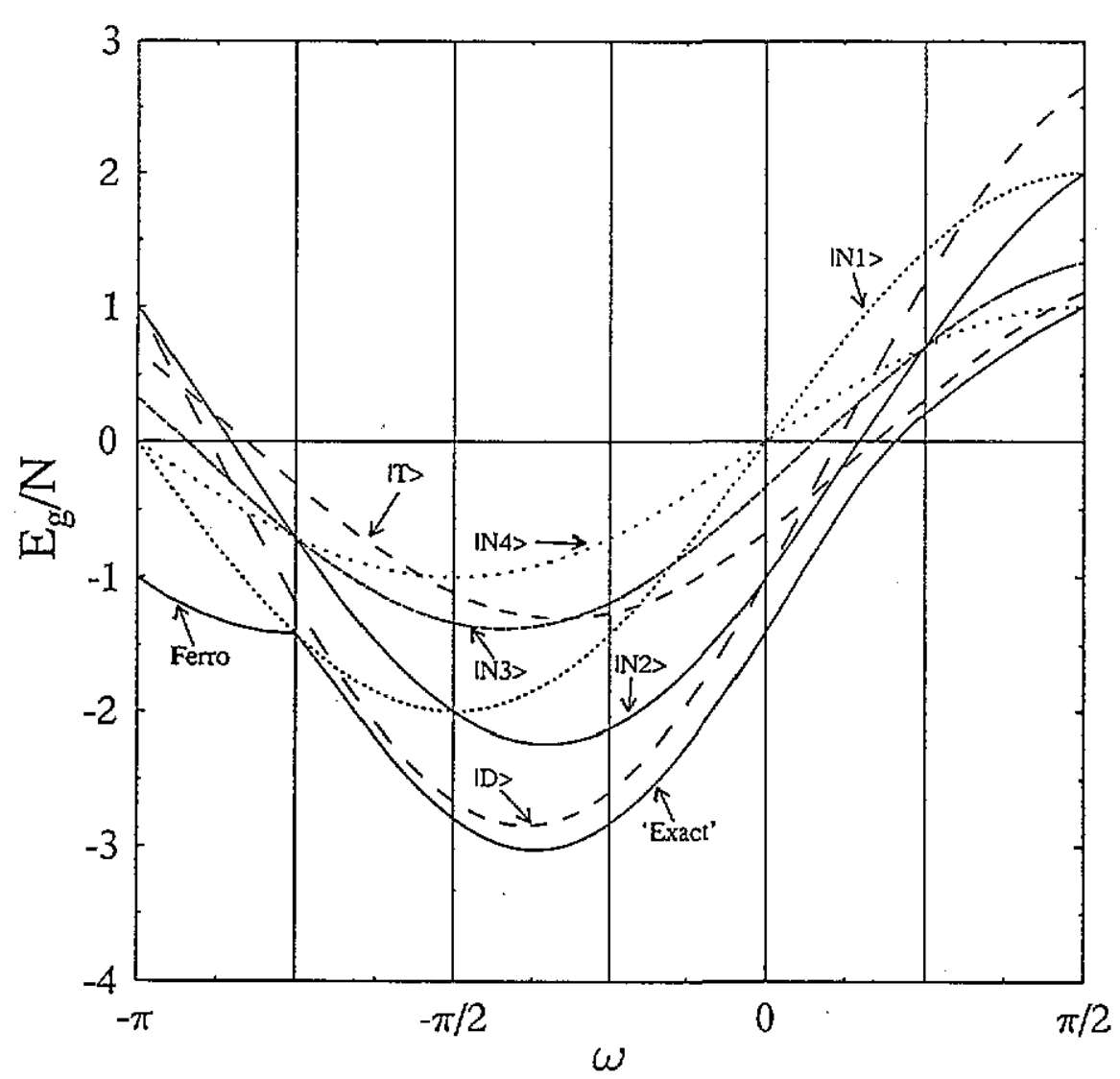

Figure 4. Expectation values of the Hamiltonian in various possible model states described in the text. The solid line marked 'exact' is as in figure 2.

on the state $|+\rangle$. Since we require that the CCM correlation operator $S$ is constructed out of mutually commuting creation operators only, this cannot be done using the $\left\{s_{i}^{ \pm}\right\}$.

For a single spin-1 atom any operator can be written as $3 \times 3$ matrix using the basis $\{1+\rangle,|0\rangle, \mid-\}\}$. There are nine independent operators which we choose as the nine matrices each of which has eight zero entries and a single non-zero entry which we take as 1 in each of the nine possible positions. If $A_{i j}$ is the $3 \times 3$ matrix with 1 on the $i$ th row and $j$ th column (i.e., in a notation with $\left\langle i^{\prime}\left|A_{i j}\right| j^{\prime}\right\rangle=\delta_{i i^{\prime}} \delta_{j j^{\prime}}$, where $i, j=1,2,3$ correspond respectively to the cases $\left.s^{z}=+1,0,-1\right)$, then we use the following notation

$$
\begin{array}{llll}
A_{11} \equiv U^{z} & A_{22} \equiv C^{z} & A_{33} \equiv D^{z} & \\
A_{12} \equiv U^{+} & A_{32} \equiv D^{+} & A_{21} \equiv U^{-} & A_{23} \equiv D^{-} \\
A_{13} \equiv U^{\mathrm{M}} & A_{31} \equiv D^{\mathrm{M}} & &
\end{array}
$$

This notation is meant to suggest 'up', 'down' and 'centre', while the superscript M denotes 'maximal' i.e. double ' $t$ ' or double '-

More details of these operators are given in appendix 2. The important feature, however, is that starting from $|0\rangle$, all other states, i.e. $|+\rangle$ and $|-\rangle$, can be created using the two 'creation' operators $U^{+}$and $D^{+}$, and furthermore these two creation operators are mutually 
commuting. Hence we can proceed to construct the correlation operator $S$ out of any combination of the operators $\left\{U_{i}^{+}, D_{i}^{+}\right\}$alone.

The next step is to rewrite the Hamiltonian, (1.1), in terms of the new operators. This is straightforward since all the usual spin operators can be expressed as $3 \times 3$ matrices and hence as linear combinations of the new operators. Again the details are given in appendix 2 . The result for the two types of term is

$$
\begin{gathered}
s_{l} \cdot s_{l+\rho}=\left(U_{l}^{z}-D_{l}^{z}\right)\left(U_{l+\rho}^{z}-D_{l+\rho}^{z}\right)+\left(U_{l}^{+}+D_{l}^{-}\right)\left(U_{l+\rho}^{-}+D_{l+\rho}^{+}\right)+\left(U_{l}^{-}+D_{l}^{+}\right)\left(U_{l+\rho}^{+}+D_{l+\rho}^{-}\right) \\
\begin{aligned}
\left(s_{l} \cdot s_{l+\rho}\right)^{2}=1 & +U_{l}^{z} D_{l+\rho}^{z}+C_{l}^{z} C_{l+\rho}^{z}+D_{l}^{z} U_{l+\rho}^{z}-\left(U_{l}^{+} D_{l+\rho}^{+}+D_{l}^{+} U_{l+\rho}^{+}+D_{l}^{-} U_{l+\rho}^{-}+U_{l}^{-} D_{l+\rho}^{-}\right) \\
& +U_{l}^{\mathrm{M}} D_{l+\rho}^{\mathrm{M}}+D_{l}^{\mathrm{M}} U_{l+\rho}^{\mathrm{M}} .
\end{aligned}
\end{gathered}
$$

Note that since the planar model state does not have two distinct sublattices there is no need to perform a notional rotation of one of them.

\subsection{The SUB2 approximation for the planar state}

Since the true ground state and the planar model state have $s_{T}^{z}=0$ we require to remain within this sector. Hence $S$ must have an equal number of $U^{+}$and $D^{+}$operators. The full SUB2 approximation involves all possible terms of this form and is written

$$
S \rightarrow S_{\mathrm{SUB} 2}=\sum_{i} \sum_{r \neq 0} \beta_{r} U_{i}^{+} D_{i+r}^{+}
$$

where $i$ runs over all sites and $r$ can be any positive or negative integer excluding 0 . Note that $r$ is not restricted to being an odd integer since the planar model state does not have two distinct sublattices. By symmetry $\beta_{-r}=\beta_{r}$. The restriction $r \neq 0$ could be dropped as $U_{i}^{+} D_{i}^{+}=0$, but we choose to keep this as an explicit restriction.

Using (2.5) and the commutators given in appendix 2 the nine transformed operators are

$\tilde{U}_{i}^{+}=U_{i}^{+}$

$\tilde{D}_{l}^{+}=D_{l}^{+}$

$\tilde{U}_{i}^{z}=U_{l}^{*}+\sum_{r \neq 0} \beta_{r} U_{l}^{+} D_{l+r}^{+}$

$\tilde{C}_{l}^{z}=C_{l}^{z}-\sum_{r \neq 0} \beta_{r}\left(U_{l}^{+} D_{l+r}^{+}+U_{l-r}^{+} D_{l}^{+}\right)$

$\tilde{D}_{l}^{z}=D_{l}^{2}+\sum_{r \neq 0} \beta_{r} D_{l}^{+} U_{l+r}^{+}$

$\tilde{U}_{l}^{-}=U_{l}^{-}+\sum_{r \neq 0} \beta_{r}\left[\left(C_{l}^{z}-U_{l}^{z}\right) D_{l+r}^{+}-U_{l+r}^{+} D_{l}^{\mathrm{M}}\right]-\sum_{r \neq 0} \sum_{s \neq 0} \beta_{r} \beta_{s}\left(U_{l}^{+} D_{l+r}^{+} D_{l+s}^{+}+D_{l}^{+} U_{l+r}^{+} D_{l+s}^{+}\right)$

$\tilde{D}_{l}^{-}=D_{l}^{-}+\sum_{r \neq 0} \beta_{r}\left[\left(C_{l}^{2}-D_{l}^{z}\right) U_{l+r}^{+}-D_{l-r}^{+} U_{l}^{\mathrm{M}}\right]-\sum_{r \neq 0} \sum_{s \neq 0} \beta_{r} \beta_{s}\left(D_{l}^{+} U_{l+r}^{+} U_{l+s}^{+}+U_{l}^{+} D_{l+r}^{+} U_{l+s}^{+}\right)$ 
$\tilde{U}_{l}^{\mathrm{M}}=U_{l}^{\mathrm{M}}+\sum_{r \neq 0} \beta_{r} U_{l}^{+} U_{l+r}^{+}$

$\tilde{D}_{l}^{\mathrm{M}}=D_{l}^{\mathrm{M}}+\sum_{r \neq 0} \beta_{r} D_{l}^{+} D_{l+r}^{+}$

The transformed Hamiltonian is obtained by replacing each of the operators on the RHS of equations (3.4) and (3.5) with the corresponding transformed operator, and then substituting into (1.1). This rather lengthy process can be shortened slightly by noting that the transformed Hamiltonian will operate on the model state and that only terms which involve either zero or two flipped spins need be retained.

The equation for the ground state energy comes from the former of these by operating with $\langle\mathrm{N} 11$ to give

$$
E_{\mathrm{g}} / N=\langle\mathrm{N} 1|\tilde{\mathcal{H}}| \mathrm{N} 1\rangle / \mathrm{N}=J_{1}\left(2 \beta_{1}\right)+J_{2}\left(2-2 \beta_{1}\right) .
$$

The set of coupled non-linear equations for the coefficients $\left\{\beta_{t}\right\}$ is obtained from the terms with two flips in $\tilde{\mathcal{H}}|\mathrm{N} 1\rangle$ by operating with $\langle\mathrm{N} 1| \sum_{i}\left(U_{i}^{-} D_{i+t}^{-}+D_{i}^{-} U_{i+t}^{-}\right)$. The result is

$K_{1} \sum_{\rho} \delta_{t \rho}+2 K_{2} \beta_{t}+K_{3} \sum_{\rho} \beta_{t+\rho}+K_{4} \sum_{s} \sum_{\rho} \beta_{s} \beta_{t+s+\rho}=0 \quad t \neq 0$

with $\beta_{0}=0$, and where

$$
\begin{aligned}
& K_{1}=J_{1}\left(1-\beta_{1}+3 \beta_{1}^{2}\right)-J_{2}\left(1-3 \beta_{1}+3 \beta_{1}^{2}\right) \\
& K_{2}=-2\left[J_{1}\left(3 \beta_{1}\right)+J_{2}\left(1-3 \beta_{1}\right)\right] \\
& K_{3}=2 J_{1} \\
& K_{4}=J_{1}-J_{2} .
\end{aligned}
$$

As was the case for the Néel model state, a simple but useful approximation, the SUB2-2 approximation, is obtained by putting all $\beta_{t}=0$ except $\beta_{ \pm 1}$. This yields

$$
J_{1}\left(1-\beta_{1}-6 \beta_{1}^{2}\right)-J_{2}\left(1+\beta_{1}-6 \beta_{1}^{2}\right)=0
$$

so that

$$
E_{\mathrm{g}} / N=\frac{1}{6}\left[11 J_{2}-J_{1}-\sqrt{24\left(J_{1}-J_{2}\right)^{2}+\left(J_{1}+J_{2}\right)^{2}}\right] .
$$

The sign of the square root has been chosen to give a physically sensible solution, as was done in the earlier papers.

\subsection{Solution of the full sUB2 planar equations}

The method of solving the coupled equations (3.9) is essentially the same as for the corresponding equations based on the Nél model state, (2.11). After Fourier transforming:

$$
\beta_{r}=\frac{1}{2 \pi} \int_{-\pi}^{\pi} \cos (r q) \beta(q) \mathrm{d} q
$$


and noting the restriction $t \neq 0$ on (3.9) we obtain the quadratic equation for $\beta(q)$ :

$$
a \beta^{2}(q)+b \beta(q)+c=0
$$

with

$$
\begin{aligned}
& a=K_{4} \cos q \\
& b=K_{2}+K_{3} \cos q
\end{aligned}
$$

and

$$
c=K_{1} \cos q-\beta_{1} K_{3}-X K_{4}
$$

where

$$
X=\sum_{s} \beta_{s} \beta_{\mathrm{s}+1}=\frac{1}{2 \pi} \int_{-\pi}^{\pi} \cos (q) \beta^{2}(q) \mathrm{d} q .
$$

In solving this quadratic equation it is necessary to take the positive sign of the square root to obtain a physically reasonable result. The choice $r=1$ in (3.12) gives a self-consistent equation for $\beta_{1}$ which is solved numerically, and hence the ground-state energy is found using (3.8). The results are shown in figure 5 . As can be seen they lie rather close to those of the SUB2-2 approximation in a region $-3 \pi / 4 \leqslant \omega \lesssim-1$.

One important difference between the full SUB2 and the SUB2-2 results is the existence of terminating points in the former. In fact there are two terminating points in this case and they occur when the term beneath the square root in (3.13) becomes negative for some value of $q$. Because of the form of the constants, $K_{1}, K_{2}, K_{3}$ and $K_{4}$, this first occurs when $q$ equals either 0 or $\pi$. The terminating point corresponding to the first of these is located at $\omega=\omega_{1}=-3 \pi / 4$, at which $\beta_{1}=0$ and $E_{g} / N=-\sqrt{2}$. This is the point at which the system becomes ferromagnetic. The one corresponding to the second of these is located at $\omega=\omega_{2}=-0.9209$, at which $\beta_{1}=-0.4264$ and $E_{g} / N=-2.7874$. These points are indicated as $P$ and $Q$ respectively in figure 5 .

The second of these terminating points is the more interesting. We note that it lies rather close in energy to the Nél SUB2 solution. Indeed at the SUB2-2 level at which only the single configuration coefficient $b_{1}$ or $\beta_{1}$ is retained, the Néel and planar solutions cross very close to $\mathrm{Q}$, at the value $\omega=-0.9050$. Such crossings are very commonly regarded in many-body theory as evidence of a phase change. The fact that the full CCM SUB2 approximation based on the planar model state terminates here is additional evidence for such a phase change. We note also that the expectation values in the uncorrelated Neel and planar model states cross at $\omega=-\pi / 2$ (see figure 4), so the additional correlations introduced by the CCM have considerably altered the predicted position of the phase change.

It is difficult to be certain at this level of approximation to which of the known and conjectured phase changes of this system, if any, this phase change corresponds. The discussion above, based on its location, would suggest rather strongly, however, that the most likely candidate is the known phase change at $\omega=-\pi / 4$ between the Haldanelike phase and the dimerized phase. Another possibility would be a transition between a 'planar quadrupolar' or 'spin-nematic' phase and a dimerized phase. This seems much less likely, however, as such a transition would have to occur in reality in the region $-3 \pi / 4<\omega \lesssim-\pi / 2$, since $\omega=-\pi / 2$ is known to have a dimerized ground state. 


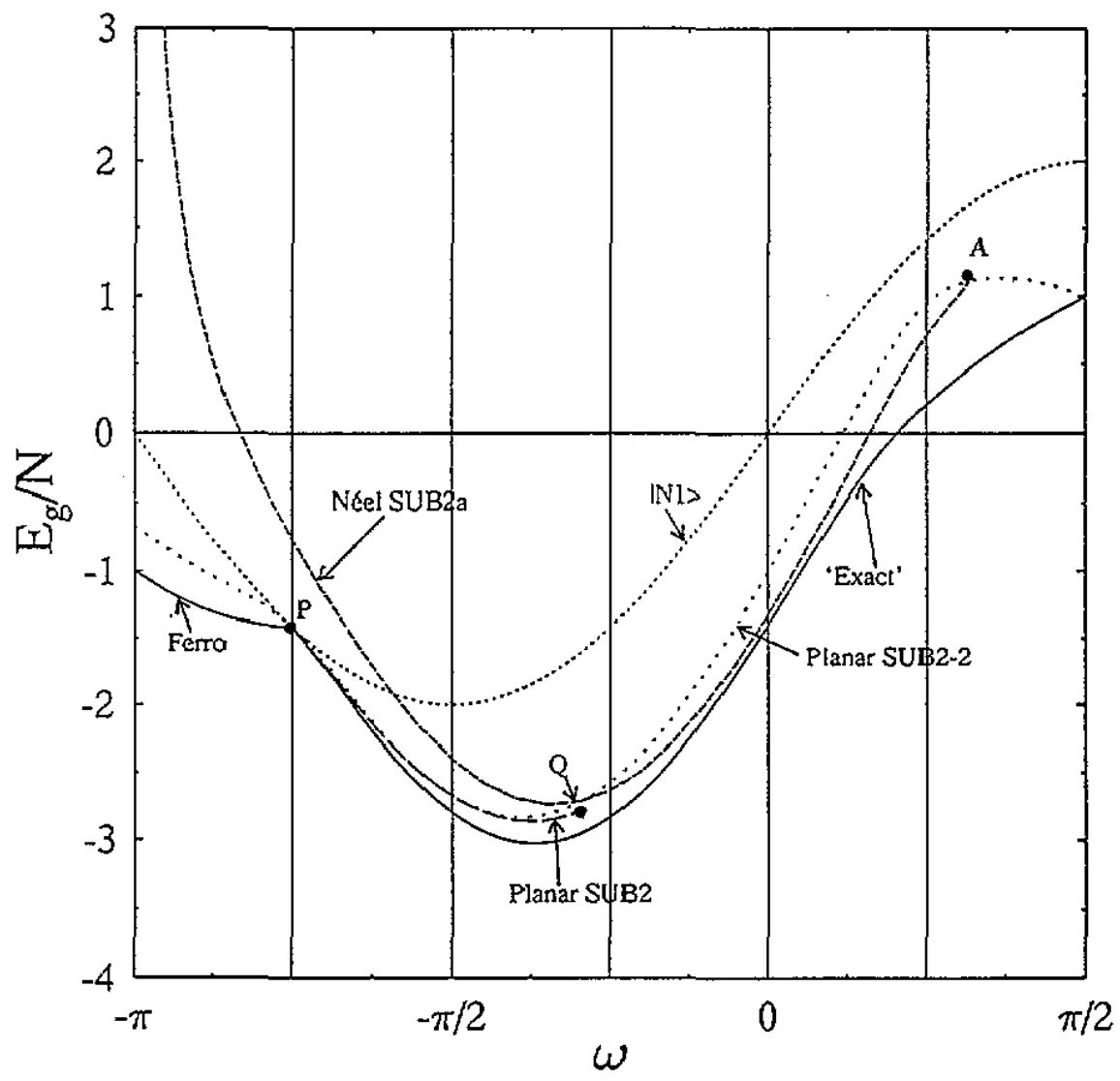

Figure 5. Ground-state energy per spin for the sUB2-2 and full suB2 approximation schemes based on the planar model state. Terminating points for the planar full suB2 scheme are at $\mathbf{P}$ $(\omega=-3 \pi / 4)$ and $\mathrm{Q}$. The full sub2a solution, based on the Néel model state, is shown for comparison. The solid line marked 'exact' is as in figure 2, and the line marked $|\mathrm{N} i\rangle$ as in figure 4.

\section{Conclusions}

This first attempt at using the CCM method to treat the Heisenberg-biquadratic spin-1 chain, with its very complex phase diagram, has produced encouraging results. We have shown how even the simple SUB2 approximation scheme, which only includes explicitly two-body terms, can yield reasonable results for the ground-state energy. As we showed for spin-1/2 systems, more accurate results are obtained by including higher-order terms in the correlation operator $S$, and this would clearly be the next step for this system also.

A new feature of the CCM as applied to this system is the use of an alternative model state and an associated new set of operators. Although the use of different model states has always been possible in principle, the difficulty until now has always been to construct the appropriate complete set of commuting creation operators. In this case the planar model state gives much better results than the Neel model state at the same level of approximation for a region of $\omega$ close to the ferromagnetic transition. Comparison of the results obtained using the two different model states and also the existence of a terminating point in both cases provides strong evidence of phase changes at various points. 
Although the CCM at the present levels of implementation are not accurate enough to give reliable numerical results for the nature and positions of the phase transitions, the ability to predict phase changes in such an $a b$ initio calculation is in itself useful. Even at the present low levels of approximation we expect that studies of the correlation functions and the elementary excitation spectrum will shed more light on these points, and we intend to do this. Including more terms in the operator $S$ would also be most interesting.

The demonstrated ability of the CCM to incorporate multi-spin correlations on top of alternative choices of reference state also encourages us to extend the present results to an even wider choice of model states. Of particular interest for the present system would be to make contact with the various valence bond analyses that have been performed for this Hamiltonian. For example, both the dimerized and the trimerized phases have been studied using a spin-wave analysis based, respectively, on the simple dimer state $|D\rangle$ and trimer state $|T\rangle$ mentioned in section 3.1, and these are obvious candidates for model states for a CCM calculation.

\section{Acknowledgment}

One of us (RFB) gratefully acknowledges the support of the Science and Engineering Research Council of Great Britain.

\section{Appendix 1. Terms in the transformed exchange interaction}

Using (2.6) the nearest-neighbour Heisenberg exchange can be written after transformation as

$$
\tilde{\boldsymbol{s}}_{l} \cdot \tilde{\boldsymbol{s}}_{l+p}=\sum_{n=-2}^{6} X_{n}
$$

where

$$
\begin{aligned}
& X_{-2}=-\frac{1}{2} s_{l}^{-} s_{l+\rho}^{-} \\
& X_{0}=-s_{l}^{z} s_{l+\rho}^{2}+s_{l}^{-} s_{l+\rho}^{z} \sum_{r} b_{r} s_{l+\rho+r}^{+}+s_{l}^{z} \sum_{r} b_{r} s_{l+r}^{+} s_{l+\rho}^{-} \\
& X_{2}=-s_{l}^{z} s_{l+\rho}^{+} \sum_{r} b_{r} s_{l+\rho+r}^{+}-s_{l}^{+} \sum_{r} b_{r} s_{l+r}^{+} s_{l+\rho}^{z}-\frac{1}{2} s_{l}^{+} s_{l+\rho}^{+}+\frac{1}{2} s_{l}^{-} s_{l+\rho}^{+} \sum_{r} \sum_{s} b_{r} b_{s} s_{l+\rho+r}^{+} s_{l+\rho+s}^{+} \\
& \quad-2 s_{l}^{z} \sum_{r} \sum_{s} b_{r} b_{s} s_{l+r^{+}}^{+} s_{l+\rho}^{z} s_{l+\rho+s}^{+}+\frac{1}{2} s_{l}^{+} \sum_{r} \sum_{s} b_{r} b_{s} s_{l+r}^{+} s_{l+s^{+}}^{+} s_{l+\rho}^{-} \\
& X_{4}=-s_{l}^{+} \sum_{r} \sum_{s} b_{r} b_{s} s_{l+r}^{+} s_{l+\rho}^{+} s_{l+\rho+s}^{+}-s_{l}^{z} \sum_{r} \sum_{r^{+}} \sum_{s} b_{r} b_{r^{\prime}} b_{s} s_{l+r^{+}}^{+} s_{l+\rho}^{+} s_{l+\rho+r^{+}}^{+} s_{l+\rho+s}^{+} \\
& \quad-s_{l}^{+} \sum_{r} \sum_{r^{\prime}} \sum_{s} b_{r} b_{r^{\prime}} b_{s} s_{l+r}^{+} s_{l+s}^{+} s_{l+\rho}^{z} s_{l+\rho+r^{\prime}}^{+}
\end{aligned}
$$

and

$$
X_{6}=-\frac{1}{2} \sum_{r} \sum_{r^{\prime}} \sum_{s} \sum_{s^{\prime}} b_{r} b_{r} b_{s} b_{s} s_{l}^{+} s_{l+r}^{+} s_{l+s}^{+} s_{l+\rho}^{+} s_{l+\rho+r^{\prime}}^{+} s_{l+\rho+s^{\prime}}^{+}
$$

$X_{6}$ is not used in this paper. 
Table A1. Products of elementary operators.

\begin{tabular}{llllllllll}
\hline & $U^{z}$ & $C^{z}$ & $D^{z}$ & $U^{+}$ & $D^{+}$ & $U^{-}$ & $D^{-}$ & $U^{\mathrm{M}}$ & $D^{\mathrm{M}}$ \\
\hline$U^{z}$ & $U^{z}$ & 0 & 0 & $U^{+}$ & 0 & 0 & 0 & $U^{\mathrm{M}}$ & 0 \\
$C^{z}$ & 0 & $C^{z}$ & 0 & 0 & 0 & $U^{-}$ & $D^{-}$ & 0 & 0 \\
$D^{z}$ & 0 & 0 & $D^{z}$ & 0 & $D^{+}$ & 0 & 0 & 0 & $D^{\mathrm{M}}$ \\
$U^{+}$ & 0 & $U^{+}$ & 0 & 0 & 0 & $U^{2}$ & $U^{\mathrm{M}}$ & 0 & 0 \\
$D^{+}$ & 0 & $D^{+}$ & 0 & 0 & 0 & $D^{\mathrm{M}}$ & $D^{z}$ & 0 & 0 \\
$U^{-}$ & $U^{-}$ & 0 & 0 & $C^{z}$ & 0 & 0 & 0 & $D^{-}$ & 0 \\
$D^{-}$ & 0 & 0 & $D^{-}$ & 0 & $C^{z}$ & 0 & 0 & 0 & $U^{-}$ \\
$U^{\mathrm{M}}$ & 0 & 0 & $U^{\mathrm{M}}$ & 0 & $U^{+}$ & 0 & 0 & 0 & $U^{z}$ \\
$D^{\mathrm{M}}$ & $D^{\mathrm{M}}$ & 0 & 0 & $D^{+}$ & 0 & 0 & 0 & $D^{z}$ & 0 \\
\hline
\end{tabular}

Table A2. Commutators of elementary operators.

\begin{tabular}{llllllllll}
\hline & $U^{z}$ & $C^{z}$ & $D^{z}$ & $U^{+}$ & $D^{+}$ & $U^{-}$ & $D^{-}$ & $U^{\mathrm{M}}$ & $D^{\mathrm{M}}$ \\
\hline$U^{z}$ & 0 & 0 & 0 & $U^{+}$ & 0 & $-U^{-}$ & 0 & $U^{\mathrm{M}}$ & $-D^{\mathrm{M}}$ \\
$C^{z}$ & 0 & 0 & 0 & $-U^{+}$ & $-D^{+}$ & $U^{-}$ & $D^{-}$ & 0 & 0 \\
$D^{z}$ & 0 & 0 & 0 & 0 & $D^{+}$ & 0 & $-D^{-}$ & $-U^{\mathrm{M}}$ & $D^{\mathrm{M}}$ \\
$U^{+}$ & $-U^{+}$ & $U^{+}$ & 0 & 0 & 0 & $U^{z}-C^{z}$ & $U^{\mathrm{M}}$ & 0 & $-D^{+}$ \\
$D^{+}$ & 0 & $D^{+}$ & $-D^{+}$ & 0 & 0 & $D^{\mathrm{M}}$ & $D^{z}-C^{z}$ & $-U^{+}$ & 0 \\
$U^{-}$ & $U^{-}$ & $-U^{-}$ & 0 & $C^{z}-U^{z}$ & $-D^{\mathrm{M}}$ & 0 & 0 & $D^{-}$ & 0 \\
$D^{-}$ & 0 & $-D^{-}$ & $D^{-}$ & $-U^{\mathrm{M}}$ & $C^{z}-D^{z}$ & 0 & 0 & 0 & $U^{-}$ \\
$U^{\mathrm{M}}$ & $-U^{\mathrm{M}}$ & 0 & $U^{\mathrm{M}}$ & 0 & $U^{+}$ & $-D^{-}$ & 0 & 0 & $U^{z}-D$ \\
$D^{\mathrm{M}}$ & $D^{\mathrm{M}}$ & 0 & $-D^{\mathrm{M}}$ & $D^{+}$ & 0 & 0 & $-U^{-}$ & $D^{z}-U^{2}$ & 0 \\
\hline
\end{tabular}

\section{Appendix 2. Properties of the planar state operators}

The nine elementary $3 \times 3$ matrices $A_{i j}$ satisfy the product law

$$
A_{i j} A_{k l}=\delta_{j k} A_{i l}
$$

and these are shown in table $A 1$, using the notation of $(3.3)$. The commutation relations are thus

$$
\left[A_{i j}, A_{k l}\right]=\delta_{j k} A_{i l}-\delta_{l i} A_{k j}
$$

which are shown in table A2.

Each of the normal spin-1 angular momentum operators can be expressed as a $3 \times 3$ matrix and hence as a linear combination of the elementary operators, e.g.

$$
s^{+}=\left(\begin{array}{ccc}
0 & \sqrt{2} & 0 \\
0 & 0 & \sqrt{2} \\
0 & 0 & 0
\end{array}\right)=\sqrt{2}\left(A_{12}+A_{23}\right)=\sqrt{2}\left(U^{+}+D^{-}\right)
$$

and similarly

$$
\begin{aligned}
& s^{-}=\sqrt{2}\left(U^{-}+D^{+}\right) \\
& s^{z}=U^{z}-D^{z} .
\end{aligned}
$$

These are the only terms needed to obtain $(3.4)$ and $(3.5)$. Note also that

$$
U^{z}+C^{z}+D^{z}=1
$$

The reverse process, in which each of the elementary matrices is expressed in terms of spin operators or products of spin operators, are also possible, e.g. $U^{\mathrm{M}}=\left(s^{+}\right)^{2} / 2$, but again these are not needed for this paper. 


\section{References}

Affick 1. Kennedy T, Lieb $\mathrm{E}$ and Tasaki 41987 Phys. Rev. Lett. 59 799-802 1988 Commun, Math. Phys, 115 477 528

Babujian H M 1982 Phys. Lett, 90A 479-82

Barber M N and Bachelor M T 1989 Phys, Rev. B 404621 6

Bethe H A 1931 2. Phys. T1 205-26

Bishop R F 1991 Theor. Chim. Acta 80 95-148

Bishop R F and Kummel $\mathrm{H} 1987$ Phys. Today 40 52 60

Bishop R F, Parkinson 1 B and Xian Y 1991a Phys. Rev. B 43 13782-5

- 19916 Phys. Rev. B $449425-43$

1992a Phys. Rev. B $46880-8$

19925 J. Phys.: Condens. Mutter 45783-94

Chubukov A V 1990 J. Phys.: Condens. Matter $21593-608$ 1991 Phys. Rey, B 43 3337-44

Fáth $\mathrm{C}$ and Solyom 31991 Phys. Rev. B 4411836 m4

س. 1993 Phys, Rev, 8 47 872-81

Haldane F D M 1983a Phys. Lett. 93A 464-8

- 19830 Phys. Rev. Lett. 50 3153m6

Kitumer A 1989 Europhys. Lett, $9815-20$ 1990 J. Phys. A: Muth, Gen, $23809-23$

Lai C K 1974 J. Math. Phys. $151675-6$

Parkinson J B 1987 J. Phys. C: Solid Stase Phys. 20 L1029-32 1988 I. Phys. C: Solid Stute Phys. 213793806

Roger M and Hetherington J H 1990 Phys. Rev, B 41 200-19

Sakai T and Takahashi M 1991 Phys. Rev. B $4313383-93$

Sutherland B 1975 Phys. Rev. B 12 3795-805

Takktajan L A 1982 Phys. Lett. 87A 479-82

$X$ Xar: Y 1993 J. Phys: Condens. Matser 57489 m502

Xiang $T$ and Gehring $G$ A 1993 Phys. Rev. B 48 303-10 